\title{
Dispersive analysis of pion-nucleon scattering and the pion-nucleon sigma term
}

\author{
Jacobo Ruiz de Elvira* ${ }^{* \dagger}$ \\ Albert Einstein Center for Fundamental Physics, \\ Institute for Theoretical Physics, University of Bern, \\ Sidlerstrasse 5, CH-3012 Bern, Switzerland \\ E-mail: elvira@itp.unibe.ch
}

\section{Martin Hoferichter}

Institute for Nuclear Theory, University of Washington, Seattle, WA 98195-1550, USA

E-mail: mhofer@uw.edu

\section{Bastian Kubis}

Helmholtz-Institut für Strahlen- und Kernphysik (Theorie) and Bethe Center for Theoretical Physics, Universität Bonn, D-53115 Bonn, Germany

E-mail: kubis@hiskp.uni-bonn.de

\section{Ulf-G. Meißner}

Helmholtz-Institut für Strahlen- und Kernphysik (Theorie) and Bethe Center for Theoretical Physics, Universität Bonn, D-53115 Bonn

Institut für Kernphysik, Institute for Advanced Simulation, and Jülich Center for Hadron Physics, Forschungszentrum Jülich, D-52425 Jülich, Germany

E-mail: meissner@hiskp.uni-bonn.de

\begin{abstract}
Pion-nucleon scattering is one of the most fundamental processes in nuclear and hadronic physics, with applications that range from the scalar couplings of the nucleon to the long-range part of two-pion-exchange potentials and three-nucleon forces in Chiral Effective Field Theory. In this talk, we show how the fruitful combination of dispersion-theoretical methods with modern highprecision data of hadronic atoms allows for a determination of pion-nucleon scattering amplitudes at low energies with an unprecedented accuracy. Special attention will be paid to the extraction of the pion-nucleon $\sigma$-term, discussing in detail the current tension with recent lattice results, the determination of the pion-pion continuum in the electromagnetic nucleon form factors, and the determination of the low-energy constants of chiral perturbation theory.
\end{abstract}

XVII International Conference on Hadron Spectroscopy and Structure - Hadron2017

25-29 September, 2017

University of Salamanca, Salamanca, Spain

\footnotetext{
*Speaker.

$\dagger$ These proceedings borrow heavily from previous conference contributions [1-3] and our original article [4].
} 


\section{Introduction}

Pion-nucleon $(\pi N)$ scattering is the simplest process involving the lightest mesons and baryons, hence allowing one to test, at low energies, the dynamical constraints imposed by chiral symmetry. The expansion around the chiral limit of QCD in terms of momenta and quark masses can be performed systematically in the framework of Chiral Perturbation Theory (ChPT) [5-7]. Nevertheless, at next-to-leading order (NLO), the $\pi N$ scattering amplitude depends on a list of lowenergy constants (LECs), which, encoding information about heavier degrees of freedom, cannot be constrained from chiral symmetry. Once determined in one process, these LECs can subsequently be used to predict others. For $\pi N$ scattering this implies applications that reach far into the domain of nuclear physics, where the same LECs that appear in the $\pi N$ amplitude govern the long-range part of the nucleon-nucleon $(N N)$ potential and the three-nucleon force.

In addition, also the partial waves for the crossed channel $\pi \pi \rightarrow \bar{N} N$ enter applications that extend beyond $\pi N$. The response of the nucleon to external currents can be analyzed via a $t$ channel dispersion relation, and depending on the quantum numbers pion-pion $(\pi \pi)$ intermediate states frequently provide the dominant contribution to the integral. In particular, for the $P$-waves, it provides a determination of the $\pi \pi$-continuum contribution to the isovector spectral functions of the nucleon electromagnetic form factors, an essential input for the analysis of the proton radius puzzle.

Finally, a further strong incentive to study pion-nucleon scattering derives from its relation to the pion-nucleon $\sigma$-term $\sigma_{\pi N}$ by means of the Cheng-Dashen low-energy theorem (LET) $[8,9]$, which requires an analytic continuation of the Born-term-subtracted isoscalar amplitude into the unphysical region. The $\sigma$-term has gathered strong interest beyond the hadron physics community in recent years, due to its relation to the scalar couplings of the nucleon that are prerequisite for a consistent interpretation of direct-detection dark matter experiments [10-12] and other searches for physics beyond the Standard Model [13-15].

\section{Roy-Steiner equations for $\pi N$ scattering}

Dispersion relations have repeatedly proven to be a powerful tool for studying processes at low energies with high precision. They are built upon very general principles such as Lorentz invariance, unitarity, crossing symmetry, and analyticity.

For $\pi \pi$ scattering, Roy equations [16] are obtained from a twice-subtracted fixed- $t$ dispersion relation, where the $t$-dependent subtraction constants are determined by means of $s \leftrightarrow t$ crossing symmetry, and performing a partial-wave expansion. This leads to a coupled system of partial-wave dispersion relations (PWDRs) for the $\pi \pi$ partial waves where the scattering lengths - the only free parameters-appear as subtraction constants. The use of Roy equations for $\pi \pi$ scattering has led to a determination of the low-energy $\pi \pi$ scattering amplitude with unprecedented accuracy $[17,18]$, which, for the first time, allowed for a precise determination of the $f_{0}(500)$ pole parameters $[19,20]$.

In the case of $\pi N$ scattering, a full system of PWDRs has to include dispersion relations for two distinct physical processes, $\pi N \rightarrow \pi N$ ( $s$-channel) and $\pi \pi \rightarrow \bar{N} N$ ( $t$-channel), and the use of $s \leftrightarrow t$ crossing symmetry will intertwine $s$ - and $t$-channel equations. Roy-Steiner (RS) equations [21] are a set of PWDR that combine the $s$ - and $t$ - channel physical region by means of hyperbolic 
dispersion relations. Subtractions are performed at the so-called subthreshold point, which proves convenient for the matching to ChPT and for the extraction of the $\sigma$-term.

The construction of a complete system of RS equations for $\pi N$ scattering has been presented in detail in [22, 23] and their solutions have been studied in [4]. These solutions are stabilized when the $S$-wave scattering lengths, known precisely from pionic atoms [4,24,25], are imposed as constraints. Final results for the $\pi N$ partial waves and subthreshold parameters are shown in [4].

\section{Consequences for the $\pi N \sigma$-term}

The Cheng-Dashen LET $[8,9]$ relates the Born-term-subtracted isoscalar amplitude evaluated at the Cheng-Dashen point $\left(v=0, t=2 M_{\pi}^{2}\right)$ to the scalar form factor of the nucleon, evaluated at momentum transfer $t=\left(p^{\prime}-p\right)^{2}=2 M_{\pi}^{2}$. In practice, this LET is often rewritten as

$$
\sigma_{\pi N}=\sigma(0)=\Sigma_{d}+\Delta_{D}-\Delta_{\sigma}-\Delta_{R},
$$

where $\Delta_{R}$ represents higher-order corrections in the chiral expansion, which are expected to be small. Here, we use the estimate $\left|\Delta_{R}\right| \lesssim 2 \mathrm{MeV}$ [26], derived from resonance saturation for the $O\left(p^{4}\right)$ LECs. $\Delta_{\sigma}$ measures the curvature in the scalar form factor, $\Delta_{D}$ parameterizes contributions to the $\pi N$ amplitude beyond the first two terms in the subthreshold expansion, and $\Sigma_{d}=F_{\pi}^{2}\left(d_{00}^{+}+2 M_{\pi}^{2} d_{01}^{+}\right)$. As shown in [27], although these corrections are large individually due to strong rescattering in the isospin- $0 \pi \pi S$-wave, they cancel to a large extent in the difference. For the numerical analysis we use $\Delta_{D}-\Delta_{\sigma}=-1.8(2) \mathrm{MeV}$ [23]. Finally, the RS results for the subthreshold parameters $d_{00}^{+}$and $d_{01}^{+}$in [4] give $\Sigma_{d}=57.9(1.9) \mathrm{MeV}$, which based on (3.1) translates immediately to [28]

$$
\sigma_{\pi N}=59.1(3.5) \mathrm{MeV}
$$

which already includes isospin-breaking corrections in the LET [28] (see also [29-31]).

This result implies a significant increase compared to the "canonical value" of $\sigma_{\pi N} \sim$ $45 \mathrm{MeV}$ [32], although already $4.2 \mathrm{MeV}$ are due to new corrections to the LET. The remaining increase of nearly $10 \mathrm{MeV}$ is dictated by the new scattering length values from pionic atom experiments. To illustrate the dependence of the $\sigma$-term on the scattering lengths used as input to the solution, we expand $\Sigma_{d}$ linearly around the central values and find

$$
\Sigma_{d}=57.9(9) \mathrm{MeV}+\sum_{I_{s}} c_{I_{s}} \Delta a_{0+}^{I_{s}}, \quad c_{1 / 2}=0.24 \mathrm{MeV}, \quad c_{3 / 2}=0.89 \mathrm{MeV}
$$

where $\Delta a_{0+}^{I_{s}}$ gives the deviation from the scattering lengths extracted from pionic atoms in units of $10^{-3} M_{\pi}^{-1}$. This linearized version produces $\Sigma_{d}=46(4) \mathrm{MeV}$ if the KH80 scattering lengths are used, in excellent agreement with the original KH80 value $\Sigma_{d}=50(7) \mathrm{MeV}$. Nevertheless, our result for the $\sigma$-term seems to be in tension with a series of recent lattice $\sigma_{\pi N}$ calculations performed near or at physical pion masses, which yield values

$$
\begin{aligned}
& \sigma_{\pi N}=38(3)(3) \mathrm{MeV} \quad(\mathrm{BMW}[33]), \quad \sigma_{\pi N}=45.9(7.4)(2.8) \mathrm{MeV} \quad(\chi \mathrm{QCD}[34]), \\
& \sigma_{\pi N}=37.2(2.6)\left(\begin{array}{l}
+4.7 \\
-2.9
\end{array}\right) \mathrm{MeV} \quad\left(\text { ETMC [35]), } \sigma_{\pi N}=35(6) \mathrm{MeV} \quad\right. \text { (RQCD [36]). }
\end{aligned}
$$


These lattice results are in significant tension with the pionic-atom spectroscopy measurements [37-39]: the linear relation between $\sigma_{\pi N}$ and the $\pi N$ scattering lengths in (3.3) can be inverted so that a given value for the $\sigma$-term imposes a constraint in the scattering-length plane [40]. The constraints corresponding to the lattice results of [33-36] compared to the bands extracted from pionic atoms reflect that while the three bands from the pionic-atom measurements nicely overlap, the lattice $\sigma$-terms favor a much smaller value of $\tilde{a}^{+}$[40]. A lattice calculation of the $\pi N$ scattering lengths may be a good way to illuminate the cause of this discrepancy.

An additional way to unravel the tension around the $\sigma$-term is to directly compare with the experimental $\pi N$ scattering data base. To this end, we computed the $\pi N$ differential cross sections $\mathrm{d} \sigma / \mathrm{d} \Omega$ using the RS solution. The scattering lengths enter as input inside the RS equations, which, in turn, allows one to generate cross-section solutions in terms of scattering-length values. At very low energies, the cross section solution can be written in a linearized form similar to (3.3) for the $\sigma$ term. Such a representation can be fit to the cross-section data base leading to the scattering-length values given in [41] and a $\pi N \sigma$-term

$$
\sigma_{\pi N}=58(5) \mathrm{MeV},
$$

directly derived from $\pi N$ scattering data. This value is fully consistent with the result from pionic atoms [28], and provides further evidence that the discrepancy with lattice calculations cannot be blamed on deficient $\pi N$ data input.

\section{Nucleon form factor spectral functions}

The $\pi \pi$ contribution to the isovector spectral functions of the nucleon electromagnetic form factors is an immediate application of the RS solution, which can be extracted from the RS $t$ channel partial waves and recent experimental results for the pion vector form factor [42-44].

The resulting spectral functions are presented in [45], where the $\pi \pi$ phase shift is consistently included in the $\pi N$ amplitude and the pion vector form factor and the potential impact of isospinviolating corrections are studied. As a further application, we also studied the $\pi \pi$ saturation of the sum rules for form-factor radii. These sum rules prove to be stable, with a resulting value for the isovector magnetic radius in good agreement with previous determinations [45]. Taking the neutron electric radius from the literature [46], we find a slight preference for a small proton radius [45], albeit the uncertainties in the spectral-function are too large to draw firm conclusions.

\section{Matching to chiral perturbation theory}

The matching to ChPT is a fundamental application of the RS solution, since it offers a unique opportunity for a systematic determination of $\pi N$ LECs [47]. One would expect the chiral expansion to work best in a kinematic region where no singularities occur, which is precisely the situation encountered in the subthreshold region. The matching is thus performed by equating the chiral expansion for the subthreshold parameters to the RS results [4].

The $\pi N$ amplitude at $O\left(p^{4}\right)$ involves 13 LECs, which correspond to the 13 subthreshold parameters that receive contributions from LECs in a fourth-order calculation. Inverting the expressions for the subthreshold parameters, we obtain the LECs and correlation coefficients given in [47]. 
At NLO only four LECs, $c_{i}$, contribute. Another four LECs, $\bar{d}_{i}$, appear at $\mathrm{N}^{2} \mathrm{LO}$, and five more, $\bar{e}_{i}$, at $\mathrm{N}^{3} \mathrm{LO}$. Comparing the different extractions up to $\mathrm{N}^{3} \mathrm{LO}$, the convergence pattern for the $c_{i}$ looks reasonably stable. In contrast, while the $\mathrm{N}^{2} \mathrm{LO} \bar{d}_{i}$ are of natural size, their values increase by nearly an order of magnitude when going to $\mathrm{N}^{3} \mathrm{LO}$ (except for $\bar{d}_{5}$ ). The origin of this behavior is due to loop corrections in some subthreshold parameters involving terms that scale with $g_{A}^{2}\left(c_{3}-c_{4}\right) \sim-16 \mathrm{GeV}^{-1}$, which are balanced by the large LECs in order to keep the subthreshold parameters at their physical values. Given such large loop corrections the errors for the LECs at a given chiral order are negligible compared to the uncertainties to be attached to the chiral expansion itself. Nevertheless, the enhancement of the $c_{i}$ can be understood from resonance saturation, which for $c_{2-4}$ is mainly due to the $\Delta(1232)$ [48-50]. The magnitude of the extracted LECs is sizably reduced when the $\Delta$ is included explicitly in a consistent power counting up to full one-loop order [51], leading to an improvement of the convergence pattern in the threshold region.

\section{Acknowledgments}

We would like to thank the organizers of the XVII International Conference on Hadron Spectroscopy and Structure for a wonderful workshop, and for the invitation to talk about our work on pion-nucleon scattering. Financial support by the DFG (SFB/TR 16, "Subnuclear Structure of Matter," CRC 110, "Symmetries and the Emergence of Structure in QCD"), the DOE (Grant No. DE-FG02-00ER41132), and the Swiss National Science Foundation is gratefully acknowledged.

\section{References}

[1] B. Kubis, J. Ruiz de Elvira, M. Hoferichter and U.-G. Meißner, PoS CD 15 (2015) 021.

[2] B. Kubis, M. Hoferichter, J. Ruiz de Elvira and U.-G. Meißner, EPJ Web Conf. 130 (2016) 01006.

[3] U.-G. Meißner, J. Ruiz de Elvira, M. Hoferichter and B. Kubis, EPJ Web Conf. 137 (2017) 01014.

[4] M. Hoferichter, J. Ruiz de Elvira, B. Kubis and U.-G. Meißner, Phys. Rept. 625 (2016) 1.

[5] S. Weinberg, Physica A 96 (1979) 327.

[6] J. Gasser and H. Leutwyler, Annals Phys. 158 (1984) 142.

[7] J. Gasser and H. Leutwyler, Nucl. Phys. B 250 (1985) 465.

[8] T. P. Cheng and R. F. Dashen, Phys. Rev. Lett. 26 (1971) 594.

[9] L. S. Brown, W. J. Pardee and R. D. Peccei, Phys. Rev. D 4 (1971) 2801.

[10] A. Bottino, F. Donato, N. Fornengo and S. Scopel, Astropart. Phys. 13 (2000) 215.

[11] J. R. Ellis, K. A. Olive and C. Savage, Phys. Rev. D 77 (2008) 065026.

[12] A. Crivellin, M. Hoferichter and M. Procura, Phys. Rev. D 89 (2014) 054021.

[13] V. Cirigliano, R. Kitano, Y. Okada and P. Tuzon, Phys. Rev. D 80 (2009) 013002.

[14] A. Crivellin, M. Hoferichter and M. Procura, Phys. Rev. D 89 (2014) 093024.

[15] J. de Vries, E. Mereghetti, C. Y. Seng and A. Walker-Loud, Phys. Lett. B 766 (2017) 254.

[16] S. M. Roy, Phys. Lett. B 36 (1971) 353. 
[17] B. Ananthanarayan, G. Colangelo, J. Gasser and H. Leutwyler, Phys. Rept. 353 (2001) 207.

[18] R. García-Martín et al., Phys. Rev. D 83 (2011) 074004.

[19] I. Caprini, G. Colangelo and H. Leutwyler, Phys. Rev. Lett. 96 (2006) 132001.

[20] R. García-Martín et al., Phys. Rev. Lett. 107 (2011) 072001.

[21] G. E. Hite and F. Steiner, Nuovo Cim. A 18 (1973) 237 [CERN-TH-1590 for app. D and E].

[22] C. Ditsche, M. Hoferichter, B. Kubis and U.-G. Meißner, JHEP 1206 (2012) 043.

[23] M. Hoferichter, C. Ditsche, B. Kubis and U.-G. Meißner, JHEP 1206 (2012) 063.

[24] V. Baru et al., Phys. Lett. B 694 (2011) 473.

[25] V. Baru et al., Nucl. Phys. A 872 (2011) 69.

[26] V. Bernard, N. Kaiser and U.-G. Meißner, Phys. Lett. B 389 (1996) 144.

[27] J. Gasser, H. Leutwyler and M. E. Sainio, Phys. Lett. B 253 (1991) 260.

[28] M. Hoferichter, J. Ruiz de Elvira, B. Kubis and U.-G. Meißner, Phys. Rev. Lett. 115 (2015) 092301.

[29] J. Gasser, M. A. Ivanov, E. Lipartia, M. Mojžiš and A. Rusetsky, Eur. Phys. J. C 26 (2002) 13.

[30] M. Hoferichter, B. Kubis and U.-G. Meißner, Phys. Lett. B 678 (2009) 65.

[31] M. Hoferichter, B. Kubis and U.-G. Meißner, Nucl. Phys. A 833 (2010) 18.

[32] J. Gasser, H. Leutwyler and M. E. Sainio, Phys. Lett. B 253 (1991) 252.

[33] S. Dürr et al., Phys. Rev. Lett. 116 (2016) 172001.

[34] Y. B. Yang, A. Alexandru, T. Draper, J. Liang and K. F. Liu, Phys. Rev. D 94 (2016) 054503.

[35] A. Abdel-Rehim et al., Phys. Rev. Lett. 116 (2016) 252001.

[36] G. S. Bali et al. [RQCD Collaboration], Phys. Rev. D 93 (2016) 094504.

[37] D. Gotta et al., Lect. Notes Phys. 745 (2008) 165.

[38] T. Strauch et al., Eur. Phys. J. A 47 (2011) 88.

[39] M. Hennebach et al., Eur. Phys. J. A 50 (2014) 190.

[40] M. Hoferichter, J. Ruiz de Elvira, B. Kubis and U.-G. Meißner, Phys. Lett. B 760 (2016) 74.

[41] J. Ruiz de Elvira, M. Hoferichter, B. Kubis and U.-G.-Meißner, arXiv:1706.01465 [hep-ph].

[42] B. Aubert et al. [BaBar Collaboration], Phys. Rev. Lett. 103 (2009) 231801.

[43] D. Babusci et al. [KLOE Collaboration], Phys. Lett. B 720 (2013) 336.

[44] M. Ablikim et al. [BESIII Collaboration], Phys. Lett. B 753 (2016) 629.

[45] M. Hoferichter et al., Eur. Phys. J. A 52 (2016) 331.

[46] K. A. Olive et al. [Particle Data Group Collaboration], Chin. Phys. C 38 (2014) 090001.

[47] M. Hoferichter, J. Ruiz de Elvira, B. Kubis and U.-G. Meißner, Phys. Rev. Lett. 115 (2015) 192301.

[48] V. Bernard, N. Kaiser and U.-G. Meißner, Int. J. Mod. Phys. E 4 (1995) 193.

[49] V. Bernard, N. Kaiser and U.-G. Meißner, Nucl. Phys. A 615 (1997) 483.

[50] T. Becher and H. Leutwyler, Eur. Phys. J. C 9 (1999) 643.

[51] D. Siemens et al., Phys. Lett. B 770 (2017) 27. 